\title{
MicroRNA-1 promotes cartilage matrix synthesis and regulates chondrocyte differentiation via post-transcriptional suppression of Ihh expression
}

\author{
TAOYU CHEN $^{1 *}$, XIANDA CHE $^{1 *}$, PENGFEI HAN ${ }^{1}$, JIANGONG LU $^{1}, \mathrm{CHUNFANG} \mathrm{WANG}^{2}$, \\ BIN LIANG $^{3}$, ZIQI HOU ${ }^{2}$, XIAOCHUN WEI ${ }^{1}$, LEI WEI ${ }^{4}$ and PENGCUI LI ${ }^{1}$
}

\begin{abstract}
${ }^{1}$ Department of Orthopedics, The Second Hospital of Shanxi Medical University, Shanxi Key Laboratory of Bone and Soft Tissue Injury Repair; ${ }^{2}$ Laboratory Animal Center of Shanxi Medical University, Taiyuan, Shanxi 030001; ${ }^{3}$ Department of Orthopedics, Fenyang Hospital Affiliated to Shanxi Medical University, Fenyang, Shanxi 032200, P.R. China;

${ }^{4}$ Department of Orthopedics, Warren Alpert Medical School of Brown University, Providence, RI 02903, USA
\end{abstract}

Received May 27, 2019; Accepted May 21, 2020

DOI: $10.3892 / \mathrm{mmr} .2020 .11296$

\begin{abstract}
Indian hedgehog signaling molecule (Ihh) is known to play critical roles in chondrogenesis and cartilage development. However, it remains largely unknown how Ihh is regulated during the process. Previous studies suggest that Ihh plays an important regulatory role in the growth and development of articular cartilage, but whether it is regulated by miRNAs is unclear. The present study aimed to investigate the effects of miR-1 on chondrocyte differentiation and matrix synthesis, and to determine whether miR-1 can regulate the Ihh signaling pathway. In the present study, the expression level of miR-1 was altered via transfection of the miR-1 mimic or inhibitor in mouse thorax chondrocytes, and the impact on chondrocyte phenotypes and Ihh expression was examined. Overexpression of miR-1 promoted the expression of the matrix synthesis-associated molecules collagen (Col)-II and aggrecan, two key components in cartilage matrix. Conversely, overexpression of miR-1 significantly downregulated the expression of chondrocyte differentiation markers Col-X and matrix metallopeptidase 13. Moreover, overexpression of miR-1 dose-dependently inhibited endogenous Ihh expression, and an association was observed between miR-1 and Ihh expression. The 3' untranslated region (UTR) of Ihh from various species contains two miR-1 binding sites. Luciferase reporter assays indicated that miR-1 post-transcriptionally
\end{abstract}

Correspondence to: Dr Pengcui Li, Department of Orthopedics, The Second Hospital of Shanxi Medical University, Shanxi Key Laboratory of Bone and Soft Tissue Injury Repair, 382 Wuyi Road, Taiyuan, Shanxi 030001, P.R. China

E-mail: 1pc1977@163.com

*Contributed equally

Key words: microRNA, miRNA-1, chondrocyte, proliferation, differentiation, Indian hedgehog suppressed Ihh expression, which was dependent on the binding of miR-1 to one of the two putative binding sites of the Ihh 3'UTR. Furthermore, via inhibition of Ihh expression, miR-1 decreased the expression of molecules downstream of Ihh in the Hedgehog signaling pathway in mouse thorax chondrocytes. This study provided new insight into the molecular mechanisms of miR-1 in regulating chondrocyte phenotypes via targeting the Ihh pathway.

\section{Introduction}

MicroRNAs (miRNAs/miRs) are a class of endogenous small single-stranded non-coding RNAs that have post-transcription regulatory functions in eukaryotes (1). Ranging from 20 to 25 nucleotides in size, miRNAs recognize the 3 ' untranslational region (3'UTR) of their target mRNAs through base pairing, and suppress the translation or promote the degradation of target mRNAs via the formation of RNA-induced silencing complexes (2-4). miRNAs are involved in a variety of physiological processes including cell proliferation, differentiation, apoptosis, energy metabolism, and various pathological conditions such as cardiac remodeling, B cell development, diabetes and allergic inflammation (5-10).

Accumulating studies have demonstrated that miRNAs are also actively involved in regulating chondrogenesis and cartilage development (11). For example, miR-410 (12), miR-29b (13) and miR-218 (14) have been identified to regulate chondrogenic differentiation of bone marrow mesenchymal stem cells. miR-1 is expressed in muscle tissues and is involved in the regulation of proliferation and differentiation of muscle tissues through targeting histone deacetylase 4 , as well as the activation of oxidative metabolism during muscle cell differentiation through the miR-1/133a-myocyte-specific enhancer factor $2 \mathrm{~A}-\Delta$ like non-canonical notch ligand 1-iodothyronine deiodinase 3 axis $(15,16)$. Knockout of skeletal muscle-specific miRNAs-1-2 in vivo resulted in the death of $50 \%$ of mice due to cardiac morphological abnormalities, electrical conduction and cell cycle disorders (17). Our previous study demonstrated that miR-1 is highly expressed in the hypertrophic zone of 
growth plate cartilage, and regulates chondrocyte phenotypes during growth plate development (18). However, the roles of miR-1 in regulating matrix synthesis and chondrocyte proliferation and differentiation have not been extensively investigated.

The Hedgehog genes were originally identified during the study of the gene mutations in Drosophila melanogaster (19). One subtype of secretory Hedgehog proteins, Indian hedgehog (Ihh), is expressed predominantly in mammalian prehypertrophic chondrocytes $(20,21)$. Ihh plays an important role in bone development and maintains bone balance before and after birth. Activation of Ihh has been reported to promote chondrocyte hypertrophy in human osteoarthritic cartilage (22) and cultured chicken chondrocytes (23). Previous studies suggest that Ihh plays an important regulatory role in the growth and development of articular cartilage (24-26), but whether it is regulated by miRNAs is unclear.

In the present study, mouse primary chondrocytes were isolated and miR-1 levels were altered via the transfection of a miR-1-specific miRNA mimic and inhibitor in chondrocytes. The expression of matrix synthesis associated molecules collagen (Col)-II and aggrecan (AGG), and chondrocyte differentiation markers Col-X and matrix metallopeptidase (MMP)-13 were evaluated upon miR-1 overexpression and inhibition in chondrocytes. Importantly, this study demonstrated that miR-1 promotes cartilage matrix synthesis and regulates the chondrocyte differentiation by the post-transcriptional suppression of the Ihh gene.

\section{Materials and methods}

miRNA mimic, inhibitor and small interfering (si)RNA oligonucleotides (oligos). The miR-1 mimic, corresponding negative control mimic (ConmiR), the miR-1 inhibitor (Anti-miR-1), control miRNA inhibitor (Control), and the siRNA oligos were purchased from Shanghai GenePharma Co., Ltd. The miRNA-1 mimics were double-stranded siRNA oligos. The sense strand of miRNA-1 mimic (5'-UGGAAUGUAAAGAAGUAUGUAU-3') consisted of 21 bases, and the antisense strand was complementary to the sense chain. The miR-1 inhibitor consisted of RNA oligos of 21 bases fully complementary to the target sequences and modified with 2 ' oxygen methyl. The siRNA oligo for knockdown of Ihh (silhh) was designed and synthesized by Shanghai GenePharma Co., Ltd., and the sequences were as follows: sense, 5'-CCUUCAGUGAUGUGCUUAUTT-3'.

Isolation and culture of primary chondrocytes. C57BL/6 mice of specific-pathogen-free-grade (male, 6-8 weeks old) were purchased and maintained in the Animal Experimental Center of Shanxi Medical University. A total of 10 mice were maintained in a specific pathogen-free (SPF) 'barrier' facility and housed under $25^{\circ} \mathrm{C}$ and humidity and alternating 12-h light and dark cycles. The mice received SPF mouse food and were provided with sterile drinking water ad libitum. All experiments involving the use of animals in this study were approved by the Ethics Committee of Shanxi Medical University. The isolation and culture of murine primary chondrocytes were conducted as previously reported (27). Briefly, mouse thoraxes were isolated and digested in PBS supplemented with $3 \mathrm{mg} / \mathrm{ml}$ collagenase D (Roche Diagnostics $\mathrm{GmbH}$ ) for $90 \mathrm{~min}$ at $37^{\circ} \mathrm{C}$ until soft tissues were peeled clean. Then, the tissues were further digested with fresh digestion medium containing $3 \mathrm{mg} / \mathrm{ml}$ collagenase $\mathrm{D}$ at $37^{\circ} \mathrm{C}$ for an additional $4 \mathrm{~h}$ with shaking. Chondrocytes were harvested after centrifugation of the suspension at $1,000 \mathrm{x} \mathrm{g}, 4^{\circ} \mathrm{C}$ for $10 \mathrm{~min}$, and cultured in Dulbecco's modified Eagle's medium/Nutrient Mixture F-12 (DMEM/F-12; 1:1 ratio mixture) medium supplemented with $10 \%, 100 \mathrm{U} / \mathrm{ml}$ of penicillin and $100 \mu \mathrm{g} / \mathrm{ml}$ of streptomycin (all purchased from Gibco; Thermo Fisher Scientific, Inc.). Chondrocytes were grown in a humidified atmosphere with $5 \% \mathrm{CO}_{2}$ at $37^{\circ} \mathrm{C}$, and the medium was changed every other day.

Chondrocyte transfection. Chondrocytes at passages 2-4 were used for transfection of the miR-1 mimic, inhibitor and siRNA. Transfection experiments were performed with Lipofectamine ${ }^{\circledR} 2000$ reagent (Invitrogen; Thermo Fisher Scientific, Inc.) following the manufacturer's protocols. Briefly, chondrocytes were seeded at $3 \times 10^{5} /$ well in 6 -well plates. The miR-1 mimic, and its inhibitor, and siRNA oligos against Ihh (40 pM) were resuspended in GenMutt buffer (Promega Corp.) and mixed with $10 \mu \mathrm{l}$ of Lipofectamine 2000 reagent for transfection of cells in a single well. At 24 or $48 \mathrm{~h}$ after the transfection, the cells were harvested for RNA isolation, western blotting and immunofluorescence.

Cell proliferation assay. Cell proliferation was detected by 5-ethynyl-2'-deoxyuridine (EdU; Cell Light EdU Apollo 567 In Vitro Imaging Kit; RiboBio) labeling of cultured cells according to a previous study (13). After permeabilization with $0.5 \%$ Triton X-100 in PBS for 10 min and washing with PBS for 3 times, the cells were subsequently stained with Apollo and DAPI at room temperature for $1 \mathrm{~h}$. The cells were observed immediately after staining under a fluorescence microscope (DM6 B; Leica Microsystems GmbH) under x40 magnification, and the percentages of positively stained cells (red) were calculated using Image Lab software 5.1 (Bio-Rad Laboratories, Inc.). A total of 300 cells in each group were counted, and three independent experiments were performed.

ELISA. The levels of Ihh (cat. no. SED116Mu; Wuhan USCN Business Co., Ltd.), MMP-13 (cat. no. SEA099Mu; Wuhan USCN Business Co., Ltd.), tissue inhibitor of metalloproteinases-1 (TIMP-1) (cat. no. SEA552Mu; Wuhan USCN Business Co., Ltd.), glycosaminoglycan (GAG) (cat. no. EK3456; Signalway Antibody, Ltd.) and Col-X (cat. no. SEC156Mu; Wuhan USCN Business Co., Ltd.) in cell culture supernatants were determined using ELISA. Chondrocytes seeded in 24-well plates at a density of 8,000 cells/well in DMEM/F-12 medium were cultured overnight, and then separately transfected with the miR-1 mimic, the miR-1 mimic control, the miR-1 inhibitor and the miR-1 inhibitor control. ELISAs were performed according to the manufacturer' s protocols. All samples were normalized according to the total protein levels and run in duplicate, and the average value was calculated.

Reverse transcription-quantitative PCR (RT-qPCR). RT-qPCR was performed as previously described (18). Total RNA was isolated from in vitro cultured chondrocytes using the RNeasy Mini kit (Qiagen GmbH). Total RNA $(1 \mu \mathrm{g})$ 
was reverse transcribed to complementary DNA (cDNA) using the iScript ${ }^{\mathrm{TM}}$ cDNA Synthesis kit (Bio-Rad Laboratories, Inc.). The cDNA (40 ng) was used to quantify the expression of the target genes by RT-qPCR, using the QuantiTect SYBR $^{\circledR}$-Green PCR kit (Qiagen $\mathrm{GmbH}$ ) with the DNA Engine Opticon ${ }^{\circledR} 2$ CFD-3220 Continuous Fluorescence Detector (MJ Research Inc.). The internal controls were 18S and U6 (cat. no. 218300; Qiagen $\mathrm{GmbH}$ ) for mRNA and miRNA, respectively (U6 primer sequence not commercially available). The stem-loop primers for miR-1 were designed and purchased from Qiagen $\mathrm{GmbH}$. The mRNA expression changes of genes, including Ihh, GLI family zinc finger (Gli)-1, smoothened, frizzled class receptor (Smo), AGG, Col-II, Col-X, Sox-9, parathyroid hormone-like hormone (PTHrP), Gli-2 and Gli-3, were quantified as described in our previous publications $(22,28)$. The thermocycling conditions were as follows: Pre-incubation of samples at $95^{\circ} \mathrm{C}$ for $5 \mathrm{~min}$, then 40 cycles of denaturation at $95^{\circ} \mathrm{C}$ for $10 \mathrm{sec}$, annealing at $55^{\circ} \mathrm{C}$ for $30 \mathrm{sec}$, and extension at $72^{\circ} \mathrm{C}$ for $30 \mathrm{sec}$. The sequences of primers used in this study are listed in Table I. Relative transcript levels were calculated by the $2^{-\Delta \Delta \mathrm{Cq}}$ method (29).

Western blotting. Total proteins were obtained after lysing in vitro-cultured chondrocytes with RIPA lysis buffer (Beyotime Institute of Biotechnology). Total protein was quantified using a bicinchoninic acid assay kit (cat. no. 23225; Pierce; Thermo Fisher Scientific, Inc.) and $30 \mu \mathrm{g}$ protein/lane was separated on a $10 \%$ SDS-PAGE gel and then transferred to a PVDF membrane (Beijing Solarbio Science \& Technology Co., Ltd.). Non-specific binding was blocked with $5 \%$ non-fat milk in Tris-buffered saline plus $0.1 \%$ TBST (cat. no. T1081; Beijing Solarbio Science \& Technology Co., Ltd) at $25^{\circ} \mathrm{C}$ for $2 \mathrm{~h}$, and then the membranes were incubated with the primary antibodies (purchased from Abcam) against Ihh (1:1,000; cat. no. ab52919), Gli-1 (1:50; cat. no. ab49314), Smo (1:100; cat. no. ab236465), Col-II (1:5,000; cat. no. ab185430), Col-X (1:300; cat. no. ab58632), and MMP-13 (1:3,000; cat. no. ab39012) overnight at $4^{\circ} \mathrm{C} . \beta$-actin (1:1,000; cat. no. ab8227; Abcam) was used as the loading control. After washing with TBST, the immobilized primary antibodies were incubated with a horseradish peroxidase-conjugated secondary goat anti-rabbit IgG antibody (1:2,000; cat. no. ab205718; Abcam) for $1 \mathrm{~h}$ at $25^{\circ} \mathrm{C}$ and visualized using the ECL kit (Thermo Fisher Scientific, Inc.). Finally, the blots were analyzed quantitatively using Image Lab software (version 5.1; Bio-Rad Laboratories, Inc.) as previously described (30).

Immunofluorescence assay. Chondrocytes cultured in vitro were rinsed in PBS three times and fixed with $4 \%$ formaldehyde in PBS for $15 \mathrm{~min}$ at room temperature. After blocking non-specific binding with $5 \%$ normal goat serum (Sigma-Aldrich; Merck KGaA) at $25^{\circ} \mathrm{C}$ for $1 \mathrm{~h}$, cells were then incubated with antibody against Col-I (1:200; cat. no. ab6308; Abcam), Col-II (1:200; cat. no. ab34712; Abcam) or Ihh (1:1,000; cat. no. ab52919; Abcam) in PBS for $8 \mathrm{~h}$ at $4^{\circ} \mathrm{C}$. After washing with PBS 3 times at room temperature, the cells were incubated with FITC-conjugated goat anti-rabbit IgG $\mathrm{H} \& \mathrm{~L}$ antibody $\left(1: 1,000\right.$; cat. no. ab6717; Abcam) in PBS at $25^{\circ} \mathrm{C}$ for $1 \mathrm{~h}$. After another three washes with PBS, the samples were incubated with $10 \mu \mathrm{g} / \mathrm{ml}$ DAPI (Beyotime Institute of Biotechnology) for $5 \mathrm{~min}$ at room temperature. After the final
Table I. Sequences of PCR primers used in this study.

\begin{tabular}{|c|c|}
\hline Gene & Primer sequence (5'-3') \\
\hline$I h h$ & $\begin{array}{l}\text { F: CCACTTCCGGGCCACATTTG } \\
\text { R: GGCCACCACATCCTCCACCA }\end{array}$ \\
\hline Gli-1 & $\begin{array}{l}\text { F: GGTCCGGATGCCCACGTGAC } \\
\text { R: TCCCGCTTGGGCTCCACTGT }\end{array}$ \\
\hline Gli-2 & $\begin{array}{l}\text { F: CAT GGT ATC CCT AGC TCC TC } \\
\text { R: GAT GGC ATC AAA GTC AAT CT }\end{array}$ \\
\hline Gli-3 & $\begin{array}{l}\text { F: CAT GAA CAG CCC TTT AAG AC } \\
\text { R: TCA TAT GTG AGG TAG CAC CA }\end{array}$ \\
\hline Smo & $\begin{array}{l}\text { F: CTCCTACTTCCACCTGCTCAC } \\
\text { R: CAAAACAAATCCCACTCACAGA }\end{array}$ \\
\hline PTHrP & $\begin{array}{l}\text { F: CAACCAGCCCACCAGAGGA } \\
\text { R: GGCGGCTGAGACCCTCCA }\end{array}$ \\
\hline Col-X & $\begin{array}{l}\text { F: GCCAGGAAAGCTGCCCCACG } \\
\text { R: GAGGTCCGGTTGGGCCTGGT }\end{array}$ \\
\hline$M M P-13$ & $\begin{array}{l}\text { F: GGACCTTCTGGTCTTCTGGC } \\
\text { R: GGATGCTTAGGGTTGGGGTC }\end{array}$ \\
\hline Col-II & $\begin{array}{l}\text { F: AAGGGACACCGAGGTTTCACTGG } \\
\text { R: GGGCCTGTTTCTCCTGAGCGT }\end{array}$ \\
\hline Aggrecan & $\begin{array}{l}\text { F: CAGTGGGATGCAGGCTGGCT } \\
\text { R: CCTCCGGCACTCGTTGGCTG }\end{array}$ \\
\hline $\operatorname{Sox} 9$ & $\begin{array}{l}\text { F: CGTGGACATCGGTGAACTGA } \\
\text { R: GGTGGCAAGTATTGGTCAAACTC }\end{array}$ \\
\hline $18 s$ & $\begin{array}{l}\text { F: CGG CTA CCA CAT CCA AGG AA } \\
\text { R: GCT GGA ATT ACC GAG GCT }\end{array}$ \\
\hline
\end{tabular}

Ihh, Indian hedgehog signaling molecule; Gli, GLI family zinc finger; Smo, smoothened, frizzled class receptor; PTHrP, parathyroid hormone-like hormone; Col, collagen; MMP-13, matrix metallopeptidase 13; Sox9, SRY-box transcription factor 9; F, forward; R, reverse.

round of three washes, the samples were mounted on slides and examined using a confocal microscope (Nikon Eclipse 80i; Nikon Corporation) under x40 magnification.

Dual-luciferase reporter assay. The online tools including TargetScan (http://www.targetscan.org) and miRanda (microrna.org) were utilized to identify target genes for miR-1. Sequence analysis indicated that miR-1 is conserved across mammalian species (miRBas accession no. MIMAT0000416; microrna.sanger.ac.uk/sequences/) and there are two miR-1 target sites in the 3'UTR of Ihh in different species (CATTCCAT and ATGACCTTCCC).

The dual-luciferase assay was employed to determine the effect of miR-1 on controlling luciferase expression, which was linked with and regulated by the 3'UTR of human Ihh gene. Three luciferase reporter plasmids containing wild-type (WT) or mutated miR-1 seed sites within the 3'UTR sequence of human Ihh gene were purchased from Sangon Biotech Co., Ltd. The 293T cells were co-transfected with a combination of $200 \mathrm{ng}$ of the wild-type-Ihh-3'UTR-Luc reporter plasmid (WT 3'UTR of human Ihh gene), or plasmid containing the 
3'UTR of Ihh mutal (miR-1 binding site 1 mutation) or the 3'UTR of Ihh muta2 (miR-1 binding site 2 mutation), or the 3'UTR of Ihh muta1 and muta2, with miR-1 mimic or control miR-NC (20 nM), and a Renilla plasmid (SV40 promoter; Sangon Biotech Co., Ltd.) using Lipofectamine 2000 reagent (Invitrogen; Thermo Fisher Scientific, Inc). After transfection for $48 \mathrm{~h}$, firefly luciferase activity was determined and adjusted by Renilla luminescence using the assay kit according to the manufacturer's instructions (Promega Corporation). Each experiment was repeated four times.

Statistical analysis. All the experiments were repeated at least three times and the results are expressed as the means \pm SD. Statistical analyses were performed using SPSS (version 19.0; IBM Corp.). Independent sample t-test was used to compare the data from two different groups. The data from multiple groups were analyzed by one-way ANOVA followed by the Turkey-Kramer multiple comparisons tests. Two-way ANOVA was used to compare the time-dependent changes in GAG. Statistical significance was set at $\mathrm{P}<0.05$.

\section{Results}

miR-1 promotes the proliferation of mouse thorax chondrocytes. First, mouse thorax chondrocytes were isolated, and the expression of matrix components Col-I and Col-II were measured by immunofluorescence. As shown in Fig. 1A, Col-I expression was not detected, while Col-II was abundantly expressed in the chondrocytes (Fig. 2C). To determine the impact of miR-1 expression on chondrocyte proliferation, the miR-1 mimic or inhibitor (anti-miR-1) was transfected into these chondrocytes to alter miR-1 expression. As validated by RT-qPCR, transfection of the miR-1 mimic significantly increased miR-1 transcription, while anti-miR-1 significantly decreased its expression, compared with the corresponding controls (Fig. 1B). EdU staining at $48 \mathrm{~h}$ post-transfection demonstrated that miR-1 levels were positively associated with chondrocyte proliferation (Fig. 1C). The miR-1 mimic almost doubled the percentage of EdU-positive chondrocytes, whereas the anti-miR-1 reduced the percentage of EdU-stained chondrocytes by $\sim 50 \%$ (Fig. 1D). Moreover, the association between miR-1 level and cell proliferation in mouse thorax chondrocytes was further validated using the CCK-8 cell proliferation assay (Fig. 1E). Furthermore, in chondrocytes at $24 \mathrm{~h}$ after transfection of the miR-1 mimic, or inhibitor, or controls, the mRNA levels of the matrix enzyme Sox-9, which is also a marker of chondrocyte proliferation, were associated with miR-1 expression levels (Fig. 1F).

miR-1 increases the expression of matrix synthesis associated molecules Col-II and AGG in mouse thorax chondrocytes. Next, the impact of miR-1 expression on matrix synthesis was examined by measuring GAG, Col-II and TIMP1 protein levels in cell culture supernatants using ELISA, and Col-II protein levels using western blotting. Whereas overexpression of miR-1 increased GAG levels in supernatant at 24 , 36 and $48 \mathrm{~h}$ post-transfection, suppression of miR-1 by anti-miR-1 significantly decreased GAG levels (Fig. 2A). Immunofluorescence staining and western blotting also indicated an association between miR-1 expression and Col-II protein expression (Fig. 2B and C). ELISA results demonstrated that a miR-1 mimic promoted the secretion of Col-II (Fig. 2D) and TIMP1 (Fig. 2E) proteins from the chondrocytes at $48 \mathrm{~h}$ after transfection, whereas transfection of anti-miR-1 reduced expression levels of these proteins in cell culture supernatants. Moreover, miR-1 promoted transcription of AGG and Col-II, as the chondrocytes transfected with the miR-1 mimic had significantly higher mRNA levels of AGG and Col-II, whereas transfection of anti-miR-1 reduced AGG and Col-II transcript levels (Fig. 2F and G).

miR-1 downregulates the expression of chondrocyte differentiation associated molecules Col-X and MMP-13 in mouse thorax chondrocytes. The impact of miR-1 expression on chondrocyte differentiation was then evaluated by quantifying protein and mRNA levels of chondrocyte differentiation related molecules, Col-X and MMP-13. Western blotting and ELISA results indicated that Col-X (Fig. 3A and B) and MMP-13 (Fig. 3A and D) protein levels decreased at $48 \mathrm{~h}$ after transfection of the miR-1 mimic and increased after transfection of anti-miR-1. In addition, as revealed by RT-qPCR assays, mRNA levels of Col-X (Fig. 3B) decreased at $24 \mathrm{~h}$ after transfection of the miR-1 mimic but increased after transfection of anti-miR-1. Therefore, an association between the expression of miR-1 and hypertrophy-associated molecules Col-X and MMP-13 was observed in mouse thorax chondrocytes.

miR-1 suppresses the expression of Ihh in mouse thorax chondrocytes. To determine the potential interaction between miR-1 function and Hedgehog signaling pathway in chondrocytes, the levels of Ihh protein and mRNA in mouse chondrocytes was quantified after transfection of the miR-1 mimic and anti-miR-1. At $48 \mathrm{~h}$ after transfection, the miR-1 mimic inhibited the expression of Ihh protein in the chondrocytes in a dose-dependent manner (Fig. 4A). Consistently, whereas the miR-1 mimic inhibited the production of Ihh protein, anti-miR-1 increased Ihh protein expression as revealed by western blotting (Fig. 4B). Immunofluorescence staining also demonstrated a role of miR-1 in downregulating Ihh expression (Fig. 4C). Moreover, the protein levels of Ihh in cell culture supernatant decreased at $48 \mathrm{~h}$ after transfection of the miR-1 mimic (Fig. 4D). Furthermore, similar findings were observed on Ihh mRNA levels at $24 \mathrm{~h}$ after transfection of the miR-1 mimic and anti-miR-1 in mouse thorax chondrocytes (Fig. 4E).

Post-transcriptional regulation of Ihh by miR-1 is dependent on the miR-1 binding site in the 3'UTR of Ihh gene. Since miR-1 regulates Ihh mRNA levels, the potential post-transcriptional inactivation of Ihh by miR-1 was explored. Sequence analysis indicated that miR-1 is conserved in different mammalian species, and there are two putative seed regions of miR-1 in the 3'UTR of the Ihh gene (positions: 5'-271-278-3' and 5'-490-498-3'), and the homologous binding sites in Ihh gene among human, mouse, chimpanzee and rat were identified (Fig. 5A). To confirm the contribution of these miR-1 binding sequences in downregulating the Ihh mRNA levels, vectors were constructed that linked the coding sequence of the luciferase gene and the 3'UTR of human Ihh gene. In these 

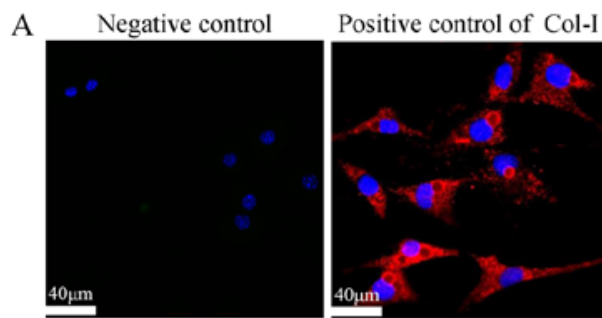

Col-I

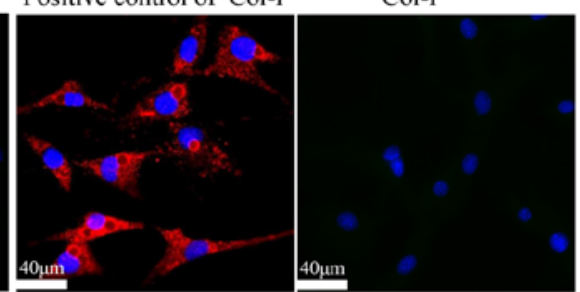

C ConmiR
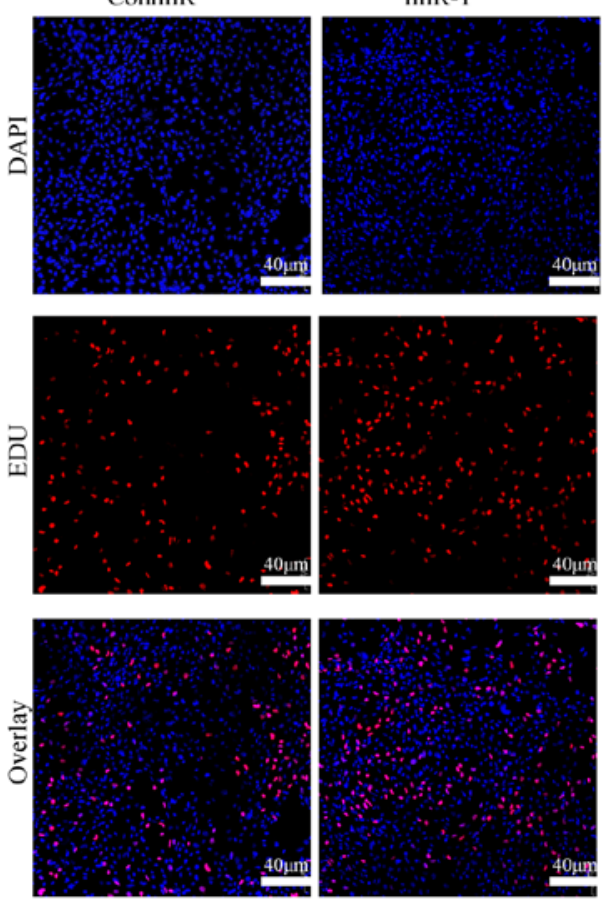

D

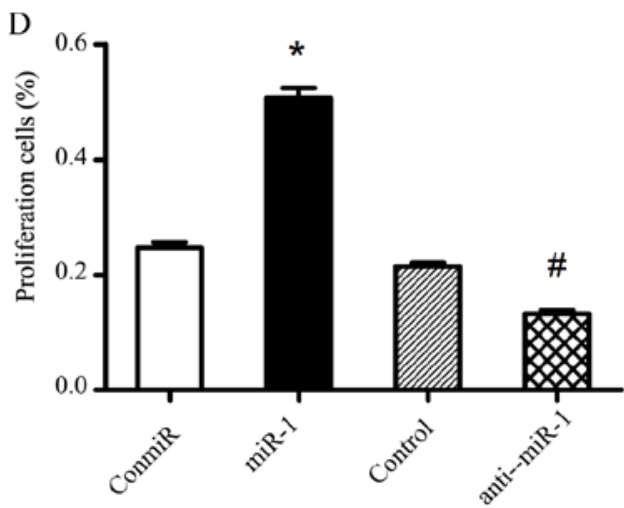

miR-1
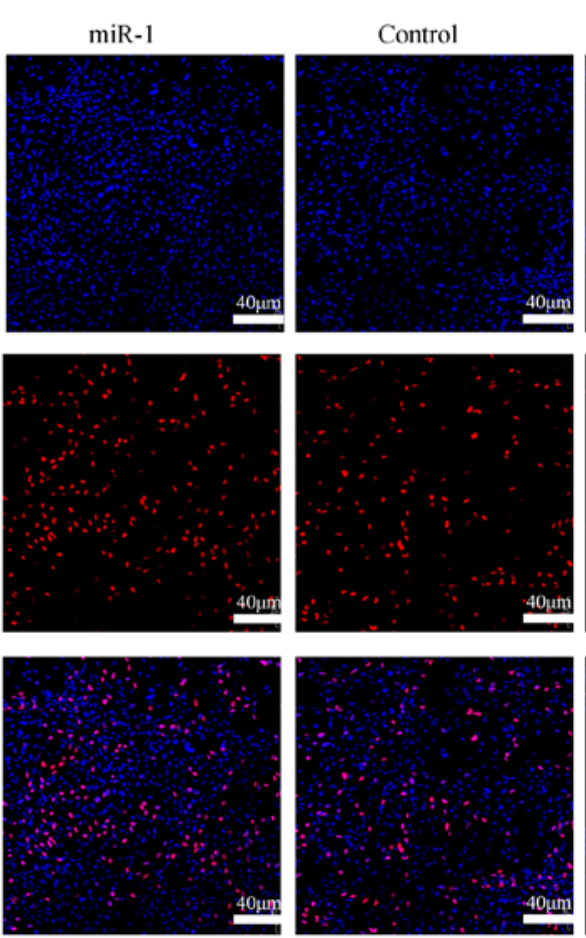

F

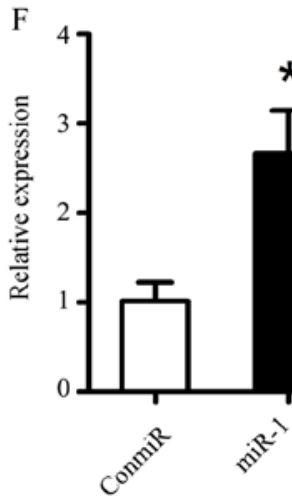

B

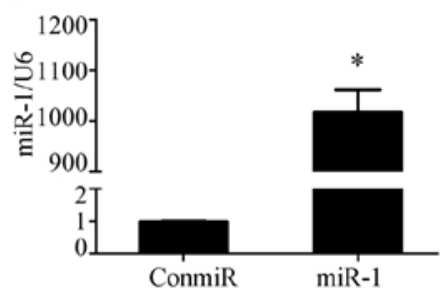

anti-miR-1
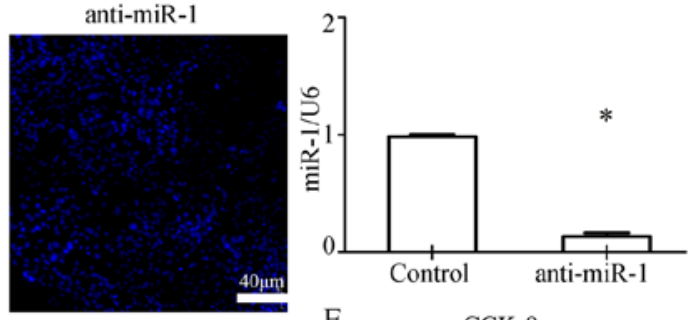

E CCK-8
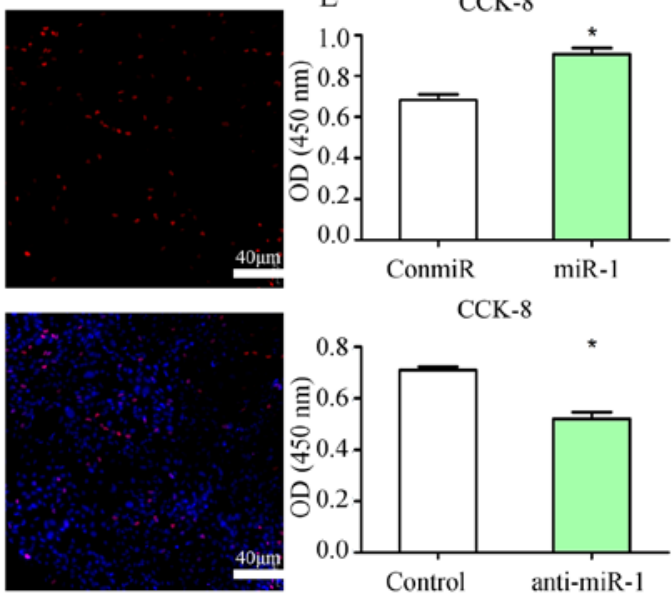

Sox-9

*

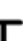

CCK-8

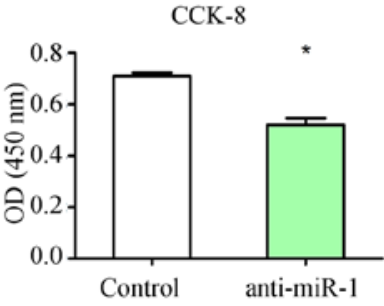

\#

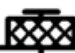

Figure 1. miR-1 promotes the proliferation of mouse thorax chondrocytes. (A) Expression of Col-I in mouse thorax chondrocytes was assessed using immunofluorescence staining. Mouse fibroblasts were used as the positive control for Col-I staining; magnification, x40; scale bars, $40 \mu \mathrm{m}$. (B) RT-qPCR results showed that transfection of the miR-1 mimic ( $40 \mathrm{pM})$ increased miR-1 levels, whereas transfection of the anti-miR-1 decreased its expression, compared with the transfection of a negative control mimic (ConmiR) or a control miRNA inhibitor (Control) in the mouse sterna chondrocytes at $24 \mathrm{~h}$ post-transfection. $\mathrm{n}=3$ for each group; ${ }^{*} \mathrm{P}<0.05$ vs. ConmiR or Control. (C and D) Cell growth was measured by EdU cell proliferation staining $48 \mathrm{~h}$ after mouse sterna chondrocytes were transfected with the miR-1 mimic (miR-1) or negative control mimic (ConmiR), and miR-1 inhibitor (anti-miR-1) or a control miRNA inhibitor (Control) at $120 \mathrm{nM}$, respectively; (C) images show the staining for EdU and DAPI, and (D) bar graph data summarizes the percentage of EdU-proliferating cells in 5 view fields per group; miR-1 stimulates chondrocyte proliferation. Scale bars, $40 \mu \mathrm{m}$; ${ }^{2} \mathrm{P}<0.05$ vs. ConmiR; ${ }^{\prime \prime} \mathrm{P}<0.05$ vs. Control. (E) Transfection of miR-1 enhanced mouse thorax chondrocyte proliferation, while transfection of anti-miR-1 inhibited proliferation, as measured by the CCK- 8 cell proliferation assay. $\mathrm{n}=5$ for each group; ${ }^{*} \mathrm{P}<0.05$ vs. ConmiR or Control. (F) miR-1 increased Sox- 9 mRNA levels, a marker for chondrocyte proliferation. Sox- 9 mRNA levels in mouse sterna chondrocytes transfected with the miR-1 mimic (miR-1), or control miRNA mimic (conmiR), and inhibitor (anti-miR-1) or control miRNA inhibitor (Control) were quantified by RT-qPCR at $24 \mathrm{~h}$ post transfection. $\mathrm{n}=3$ for each group; ${ }^{*} \mathrm{P}<0.05$ vs. ConmiR; " $\mathrm{P}<0.05$ vs. Control. Col, collagen; Sox9, SRY-box transcription factor 9; RT-qPCR, reverse transcription-quantitative PCR; miR/miRNA, microRNA.

vectors, the 3'UTR of human Ihh gene included either two WT miR-1 seed regions, one mutated miR-1 seed region, or two mutated seed regions. Luciferase reporter assays indicated that a miR-1 mimic suppressed luciferase activity of the WT
Ihh-3'UTR reporter containing two binding sites, and this suppression was abolished when expressing the Ihh-3'UTR reporter containing mutations of two putative miR-1 binding sites (mutated-1 and 2) or a single mutation of the miR-1 
A
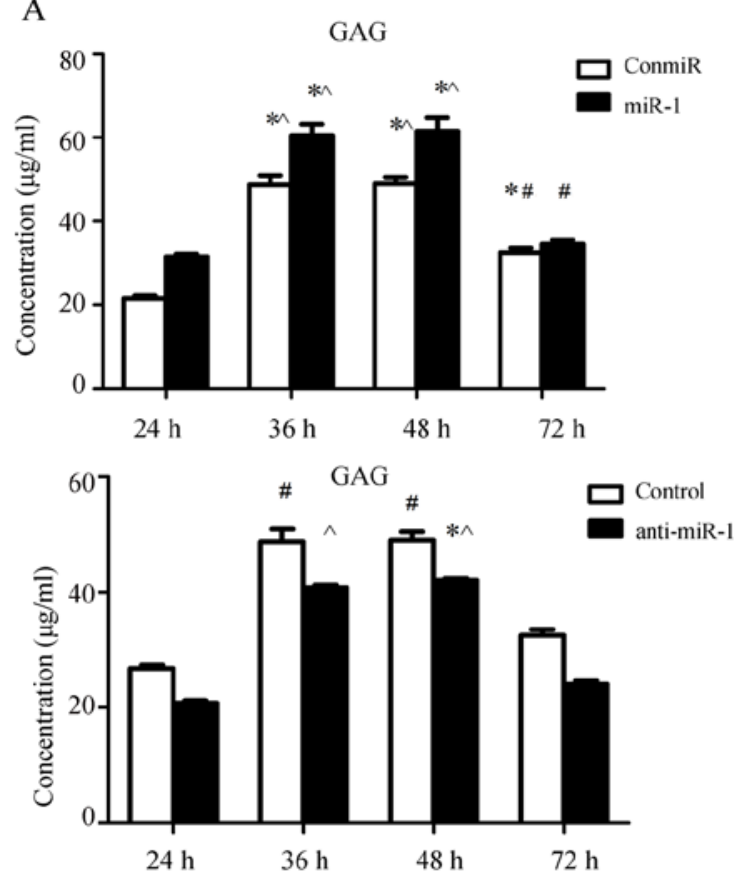

D

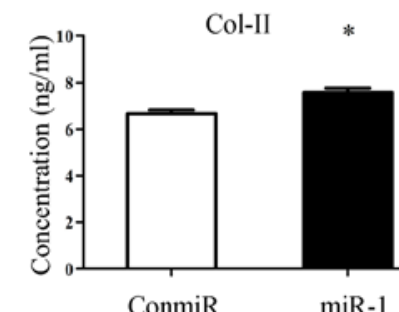

$\mathrm{E}$

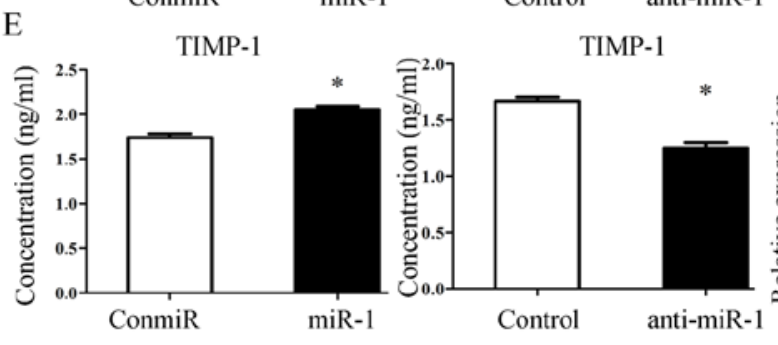

B
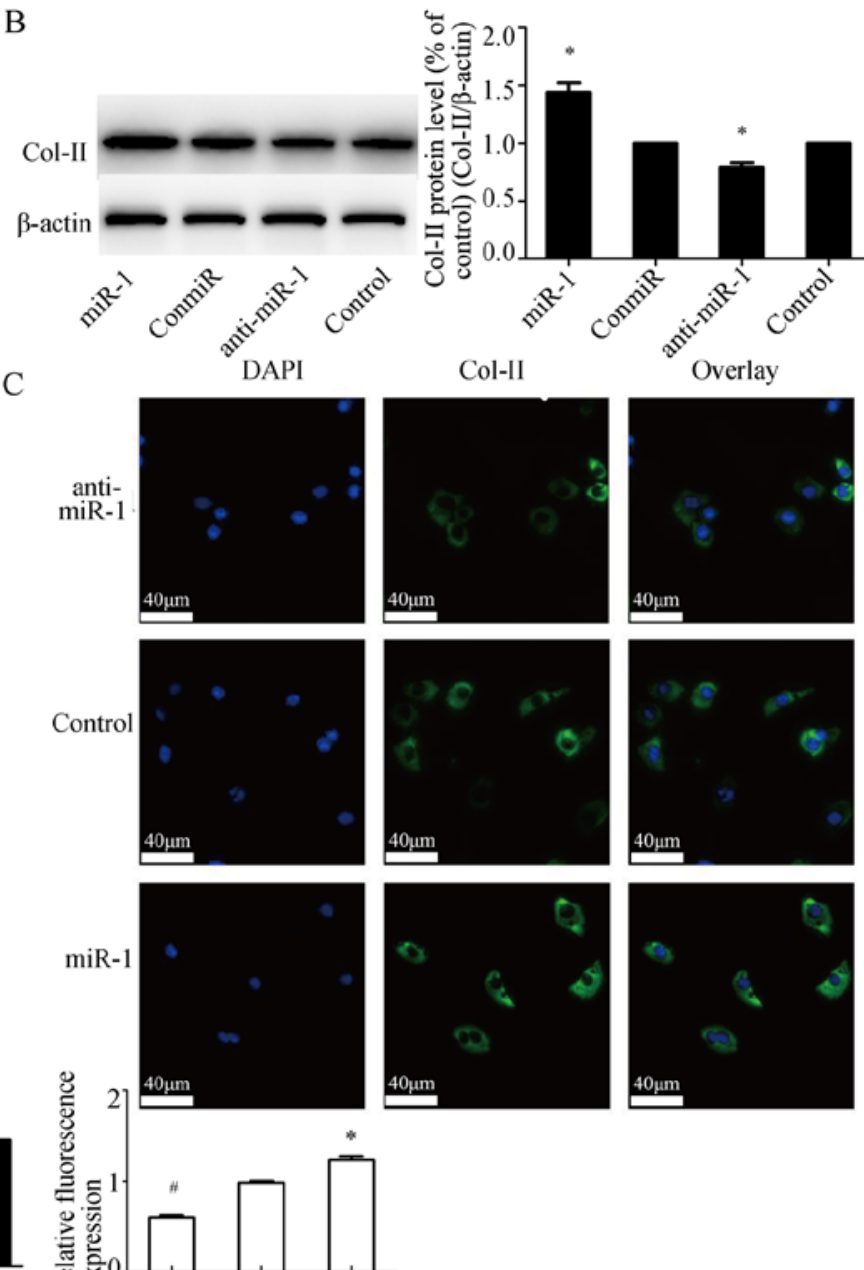

G
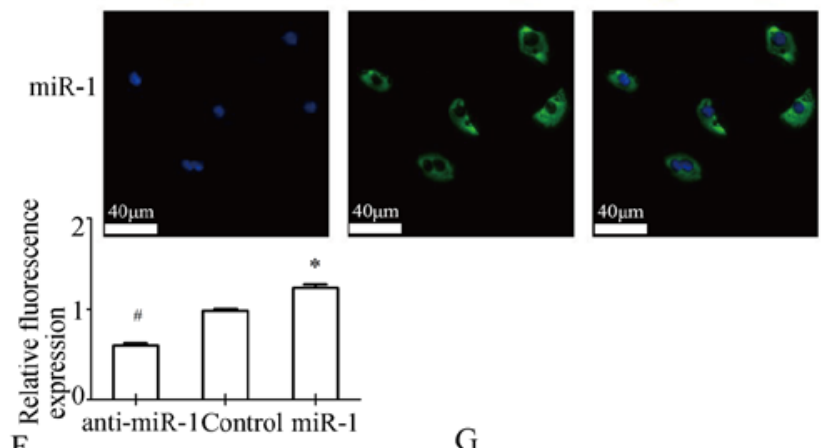

F $\quad$ AGG
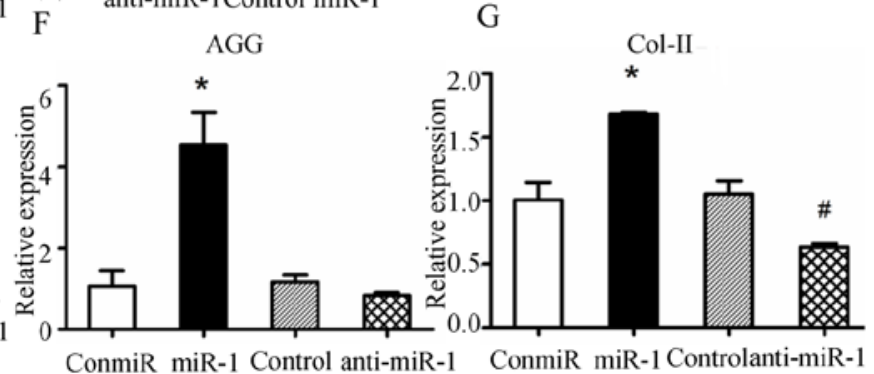

Figure 2. miR-1 increases the expression of matrix synthesis-associated molecules Col-II and AGG in mouse thorax chondrocytes. (A) Overexpression of miR-1 by transfection of the miR-1 mimic increased GAG in cell culture supernatant at 24,36 and $48 \mathrm{~h}$ post-transfection in mouse thorax chondrocytes. ${ }^{*} \mathrm{P}<0.05$ vs. the same group at $24 \mathrm{~h} ;{ }^{\#} \mathrm{P}<0.05$ vs. the same group at $48 \mathrm{~h} ;{ }^{\wedge} \mathrm{P}<0.05$ vs. the same group at $72 \mathrm{~h}$. (B) Levels of Col-II were analyzed by western blotting $48 \mathrm{~h}$ after miR-1 mimic or anti-miR transfection. The relative protein expression was normalized to $\beta$-actin expression. ${ }^{*} \mathrm{P}<0.05 \mathrm{vs}$. ConmiR or Control group. (C) Col-II expression in the chondrocytes was assessed using immunofluorescence. Magnification, $\mathrm{x} 40$; scale bars, $40 \mu \mathrm{m}$. Image $\mathrm{J}$ was used to perform semi-quantitative analysis of fluorescence intensity. ${ }^{*} \mathrm{P}<0.05$ vs. Control group. ${ }^{\#} \mathrm{P}<0.05$ vs. Control group. ELISA results demonstrated secreted (D) Col-II and (E) TIMP-1 proteins in cell culture supernatants at $48 \mathrm{~h}$ after transfection of the miR-1 mimic or inhibitor in the chondrocytes. RT-qPCR results show the relative mRNA levels of (F) AGG and (G) Col-II in the mouse chondrocytes with the indicated transfection. $\mathrm{n}=3$ for each group; ${ }^{*} \mathrm{P}<0.05$ vs. ConmiR group; ${ }^{\#} \mathrm{P}<0.05$ vs. Control group. miR/miRNA, microRNA; Col, collagen; GAG, glycosaminoglycan; TIMP, tissue inhibitor of metalloproteinases 1; AGG, aggrecan; RT-qPCR, reverse transcription-quantitative PCR.

putative binding site 2 (mutated-2; Fig. 5C). These results suggested that the miR-1 binding site 2 contributes to the suppression of Ihh expression by miR-1.

miR-1 decreases the expression of Ihh downstream molecules in the Hedgehog signaling pathway in mouse thorax chondrocytes. To further validate the post-transcriptional regulation of miR-1 on the Ihh gene, the impact of altered miR-1 expression on known genes downstream of Ihh in the
Hedgehog signaling pathway was examined in mouse thorax chondrocytes. Overexpression of miR-1 with the miR-1 mimic decreased the mRNA levels of Gli-1, Gli-2, Gli-3, Smo and PTHrP, while knockdown of miR-1 by anti-miR-1 transfection significantly increased the expression of these Ihh downstream genes, compared with the control miRNAs (Fig. 6A). To determine whether the regulation of these genes required Ihh, the mRNA levels of Gli-1, Gli-2, Gli-3, Smo and PTHrP was quantified in mouse chondrocytes with knockdown of miR-1 

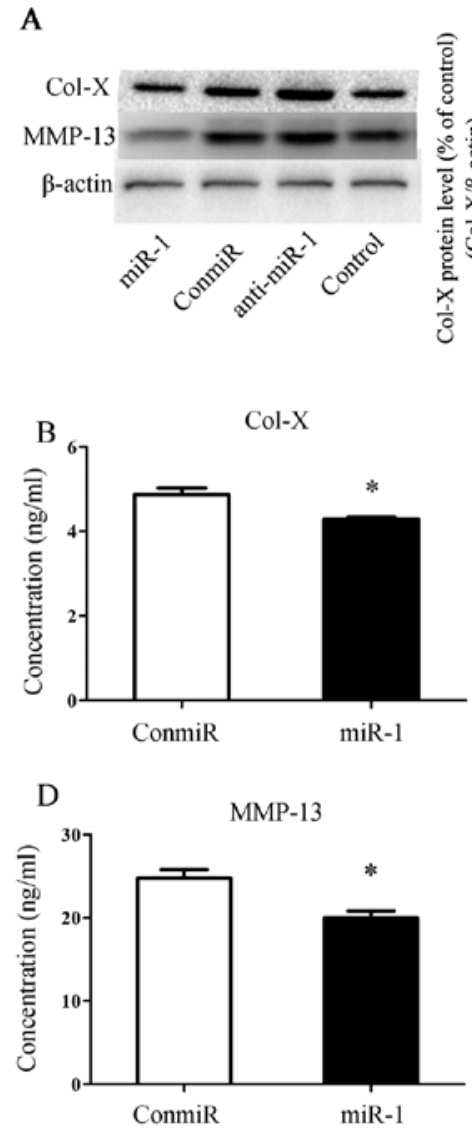

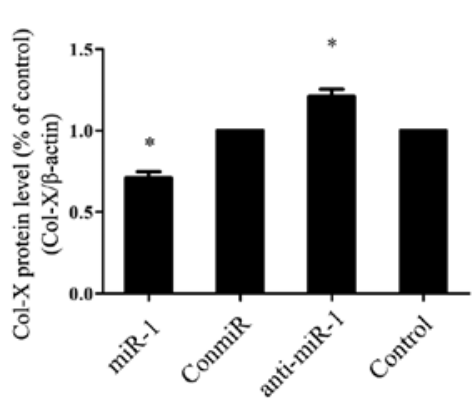

Col-X
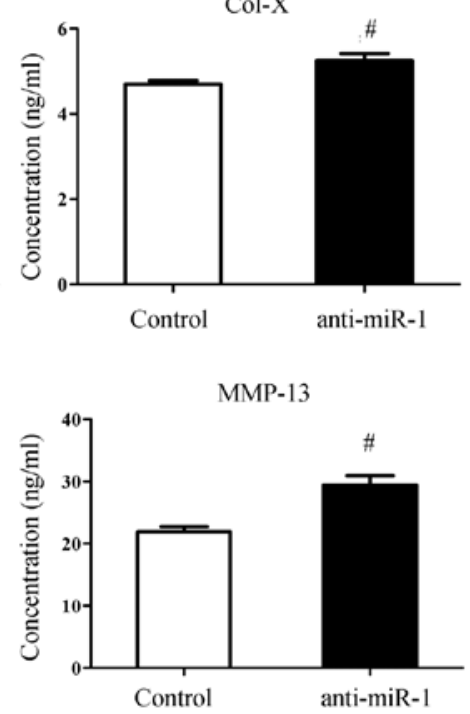

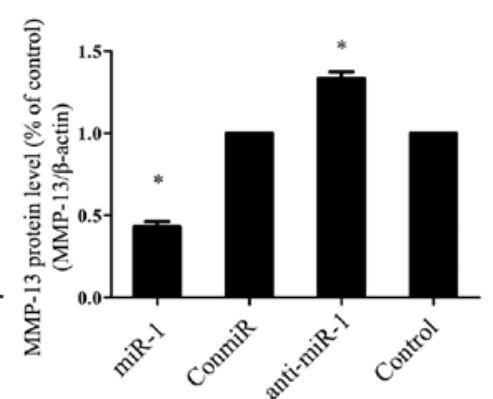

C

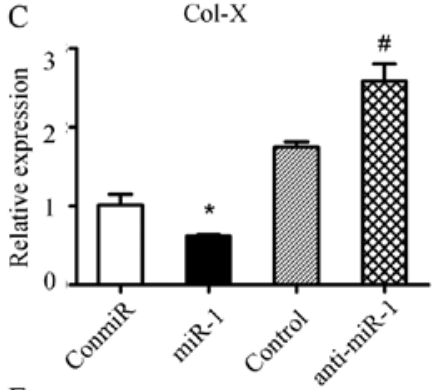

E

MMP-13

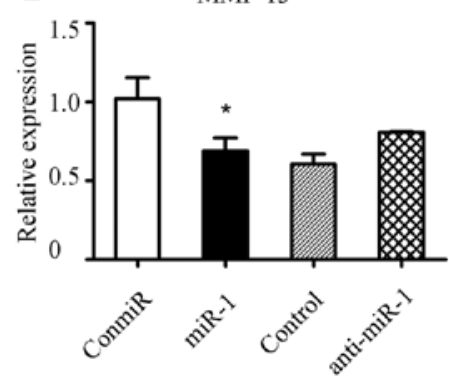

Figure 3. miR-1 downregulates the expression of chondrocyte differentiation-associated molecules Col-X and MMP-13 in mouse thorax chondrocytes. (A) Levels of Col-X and MMP-13 were analyzed by western blotting $48 \mathrm{~h}$ after miR-1 mimic or anti-miR transfection. The relative protein expression was normalized to $\beta$-actin expression. ${ }^{*} \mathrm{P}<0.05$ vs. ConmiR or Control group. (B-E) Mouse thorax chondrocytes were transfected with the miR-1 mimic (miR-1), or control miRNA (ConmiR), or the miR-1 inhibitor (anti-miR-1) or control miRNA inhibitor (Control), respectively. (B) Col-X and (D) MMP-13 protein levels in the cell culture supernatants were determined using ELISA at $48 \mathrm{~h}$ after transfection. $\mathrm{n}=3$ for each group; ${ }^{*} \mathrm{P}<0.05 \mathrm{vs}$. ConmiR group; ${ }^{\#} \mathrm{P}<0.05 \mathrm{vs}$. Control. mRNA levels of (C) Col-X and (E) MMP-13 in the chondrocytes were quantitated by RT-qPCR at $48 \mathrm{~h}$ after the indicated transfection. $\mathrm{n}=3$ for each group; ${ }^{*} \mathrm{P}<0.05$ vs. ConmiR group; ${ }^{\text {P }}<0.05$ vs. Control group. miR/miRNA, microRNA; Col, collagen; MMP-13, matrix metallopeptidase; RT-qPCR, reverse transcription-quantitative PCR.

(anti-miR-1) and knockdown of both miR-1 (anti-miR-1) and Ihh (siIhh; Fig. 6B). Results of RT-qPCR assays demonstrated that anti-miR-1 significantly increased Gli-1, Gli-2, Gli-3, Smo and PTHrP mRNAs, and knockdown of Ihh decreased Smo and Gli-1 expressions (Fig. 6B), but knockdown of Ihh completely abrogated the effects of anti-miR-1 on mRNA levels of these genes (Fig. 6C). Moreover, western blotting results showed that the Gli-1 and Smo protein levels were significantly increased in chondrocytes transfected with anti-miR-1, and Ihh knockdown abolished the effect of anti-miR-1 (Fig. 6D). Collectively, these data indicated that miR-1 regulated the expression of downstream genes in the Hedgehog signaling pathway via the post-transcriptional inactivation of Ihh.

\section{Discussion}

The signaling pathways regulating chondrocyte hypertrophy are not completely understood. Significant changes in the extracellular matrix have been observed after hypertrophy, such as downregulation of Col-II, and upregulation of MMP-13 and Col-X (31). When Ihh expression was suppressed, the expression of Gli-1, Gli-2, type X collagen and MMP-13 was decreased and the expression of AGG and type II collagen was increased (32). However, the mechanism by which miRNAs are involved in the regulation of extracellular matrix metabolism during chondrocyte hypertrophy is not fully understood. miR-1 plays an important role in muscle cell proliferation and differentiation $(15,16)$. The effects of miR-1 on the growth and development of growth plate have been systematically studied by our group (18). In the present study it was shown that miR-1 enhanced the expression of the matrix synthesis-associated molecules Col-II, GAG, TIMP1 and AGG, and inhibited the expression of chondrocyte differentiation markers Col-X and MMP-13 in mouse thorax chondrocytes. Notably, it was demonstrated that miR-1 post-transcriptionally suppressed Ihh expression via the targeting of one of the putative binding sites in the 3'UTR of the Ihh gene, and regulated Hedgehog signaling downstream genes.

Our previous results showed that miR-1 was expressed in the articular cartilage and growth plate in addition to muscle tissues of various species (18). Expression levels of miR-1 in different regions of chicken tibia growth plates were different as revealed by various experimental methods. In particular, 
A
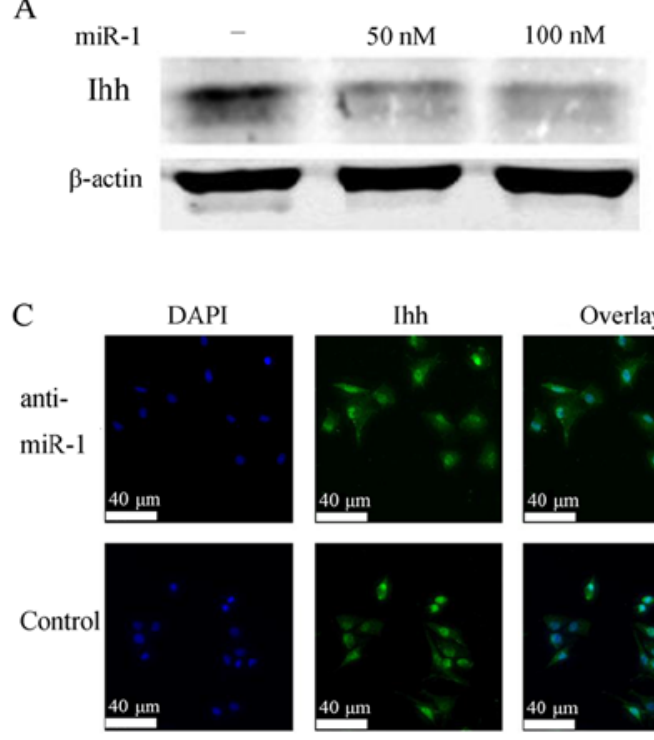

miR-1

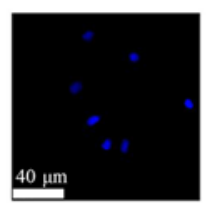

Ihh
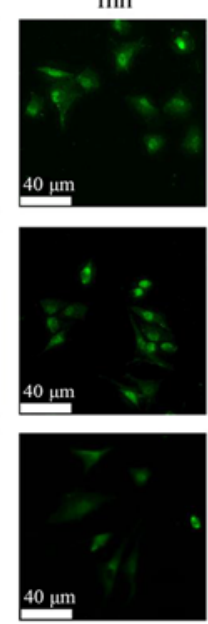

B
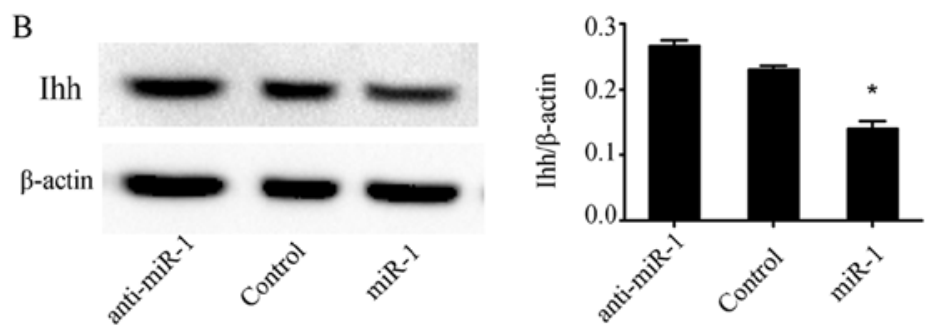

D
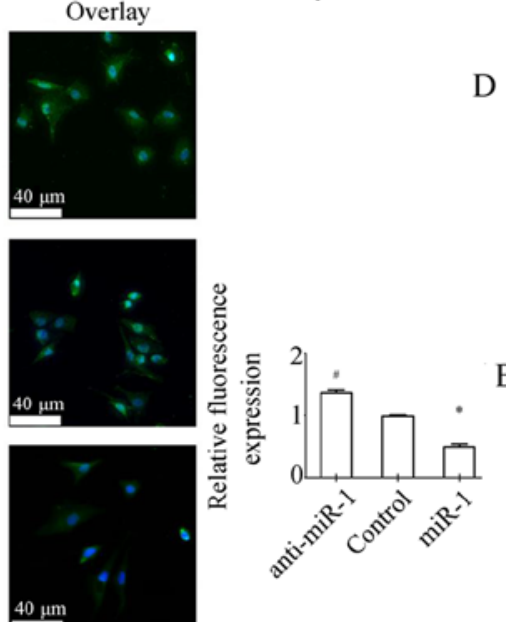

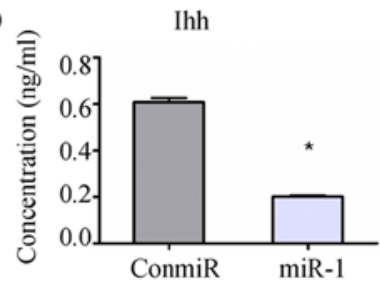

Ihh

E

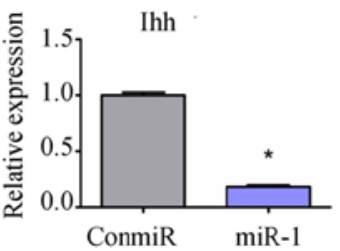

Ihh

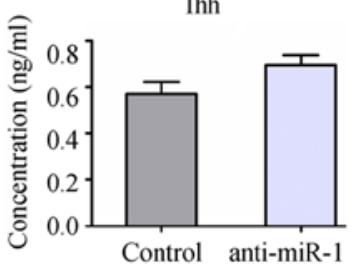

Ihh

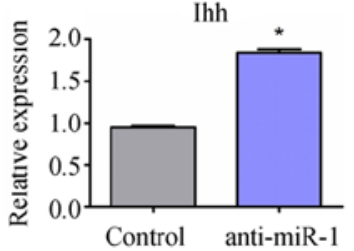

Figure 4. miR-1 inhibits Ihh expression in mouse thorax chondrocytes. (A) miR-1 mimic (miR-1) downregulated Ihh protein expression in mouse thorax chondrocytes in a dose-dependent manner. Chondrocytes were transfected with a miR-1 mimic (miR-1) at 50 and $100 \mathrm{nM}$. Samples were collected and were subjected to western blot analysis $48 \mathrm{~h}$ after transfection. (B) Thorax chondrocytes were transfected with the miR-1 mimic (miR-1), miR-1 inhibitor (anti-miR-1) and negative control mimic (ConmiR). At $48 \mathrm{~h}$ after transfection, the Ihh protein levels were determined by western blot assays. The representative images are from one of three independent experiments with similar results. Band intensities were quantified with normalization to $\beta$-actin. ${ }^{*} \mathrm{P}<0.05$ vs. Control group. (C) Representative images of immunofluorescence staining show Ihh expression in the chondrocytes with indicated transfection. Magnification, $\mathrm{x} 40$; scale bars, $40 \mu \mathrm{m}$. ImageJ was used to perform semi-quantitative analysis of fluorescence intensity. * P<0.05 vs. Control group. (D) ELISA results show secreted Ihh protein in cell culture supernatants at $48 \mathrm{~h}$ after the indicated transfection in the chondrocytes. ${ }^{*} \mathrm{P}<0.05$ vs. ConmiR or Control group. ${ }^{\#} \mathrm{P}<0.05$ vs. Control group. (E) mRNA levels of Ihh were quantified by RT-qPCR at $24 \mathrm{~h}$ post-transfection. $\mathrm{n}=3$ for each group; ${ }^{*} \mathrm{P}<0.05 \mathrm{vs}$. ConmiR or Control group. miR/miRNA, microRNA; Ihh, Indian hedgehog signaling molecule; RT-qPCR, reverse transcription-quantitative PCR.

the pre-hypertrophic and hypertrophic zones had higher miR-1 expression than proliferative zone, which implies a correlation between miR-1 expression levels and Ihh $(18,24)$. Additionally, miR-1 has been identified as an miRNA involved in the regulation of chondrocyte phenotypes during the late stages of differentiation, and its expression was most repressed upon hypertrophic differentiation (33). Consistent with those findings, the current study demonstrated that miR-1 promoted chondrocyte proliferation. Increased EdU-positive cells and higher OD values in the CCK-8 assay were observed when miR-1 was overexpressed in chondrocytes by the miR-1 mimic transfection, whereas knockdown of miR-1 by inhibitor transfection significantly reduced chondrocyte proliferation. Moreover, overexpression of miR-1 promoted the mRNA expression of Sox-9, a specific marker of chondrocyte proliferation (34), which further substantiates the proliferative role of miR-1 in mouse primary chondrocytes. Overexpression of miR-1 increased GAG levels in the supernatant at 24, 36 and $48 \mathrm{~h}$ post-transfection, and the reason for a lack of a significant change of GAG at $72 \mathrm{~h}$ upon miR-1 post-transfection may be due to the transient transfection and expression of miR-1. The increased expression of Col-II and AGG in mouse thorax chondrocytes supported the hypothesis that miR-1 promotes matrix synthesis metabolism.

Ihh is a secretory protein that belongs to the Hedgehog signaling family. It is mainly expressed in mammalian pre-hypertrophy chondrocytes, and regulates the growth and differentiation of growth plate chondrocytes by regulating their hypertrophy $(20,21,24)$. Western blotting results demonstrated that overexpression of miR-1 inhibited Ihh protein expression, whereas inhibition of miR-1 displayed the opposite effect. The negative correlation between miR-1 and Ihh expression is consistent with the notion that miRNAs control the translation and/or mRNA degradation of target genes (9). As the IHH-PTHrP axis participates in the maintenance of articular cartilage and regulates the proliferation and differentiation of articular chondrocytes (24), the present data suggested that inhibition of Ihh and the Hedgehog signaling pathway contributed to the role of miR-1 in stimulating chondrocyte proliferation.

It has been demonstrated that miRNAs act via the 3'UTRs of targeted transcripts (9). Sequence analysis indicated that miR-1 is conserved in mammalian species (miRBas accession no. MIMAT0000416; microrna.sanger.ac.uk/sequences/) and homologous binding sites in the Ihh gene between multiple species were identified. Within the Ihh 3'UTR two miR-1 binding sites were identified. The present data demonstrated that the miR-1 mimic inhibited luciferase activity that was linked with the post-transcriptional suppression of miR-1, when the luciferase reporter plasmid harboring the two WT binding sites of Ihh 3'UTR was used. Thus, a decrease of luciferase activity by miR-1 expression indicated the post-transcriptional 

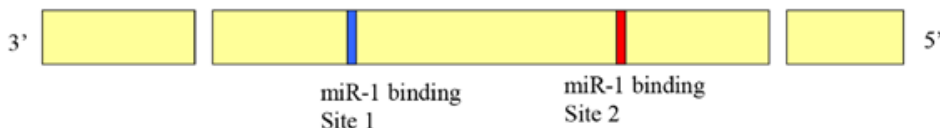

Mmu-miR-1:3'UAUGUAUGAAGAAAUGUAAGGU5' 3' UAUGUAUGAAGAAAUGUAAGGU5'

|| || | | || | ||

Mmu : $\quad 5$ ACACGCAUUCCAUCUG...... ......AACAUGACCUUCCCUG.......

Hsa : 5'UGGGGAGGGCCCAUUCCAUCCA....... .......GGUAUGACAUUCCUCC.......

Chimp: $\quad$ 'U'UGGGAGGGCCCAUUCCAUCUG.............GGUAUGACAUUCCUCC ...... 3'

Rat: $\quad$ 5'CAGGAAGGACCCAUUCCGUCUG...... .......AACAUGACCUUCCCUG.......3'

B

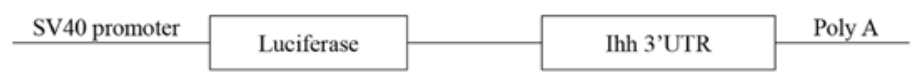

Mmu-miR-1 3'.....UAUGUAUGAAGAAAUGUAAGGU......5

\begin{tabular}{|c|c|c|}
\hline Ihh-3'UTR & $5, \ldots .$. ACGCAUUCCAU ...... & UGACCUUCC.......3' \\
\hline Ihh-3'UTR-muta(1) & 5'.......ACACGAAGUGCC. & UGACCUUCC.......3' \\
\hline Ihh-3'UTR-muta(2) & 5'.......ACGCAUUCCAU...... & GGUGCUAGA ........3' \\
\hline Ihh-3'UTR-muta(1+2) & 5 '......ACACCAAGUGCC & GGUGCUAGA ........3' \\
\hline
\end{tabular}

$\mathrm{C}$

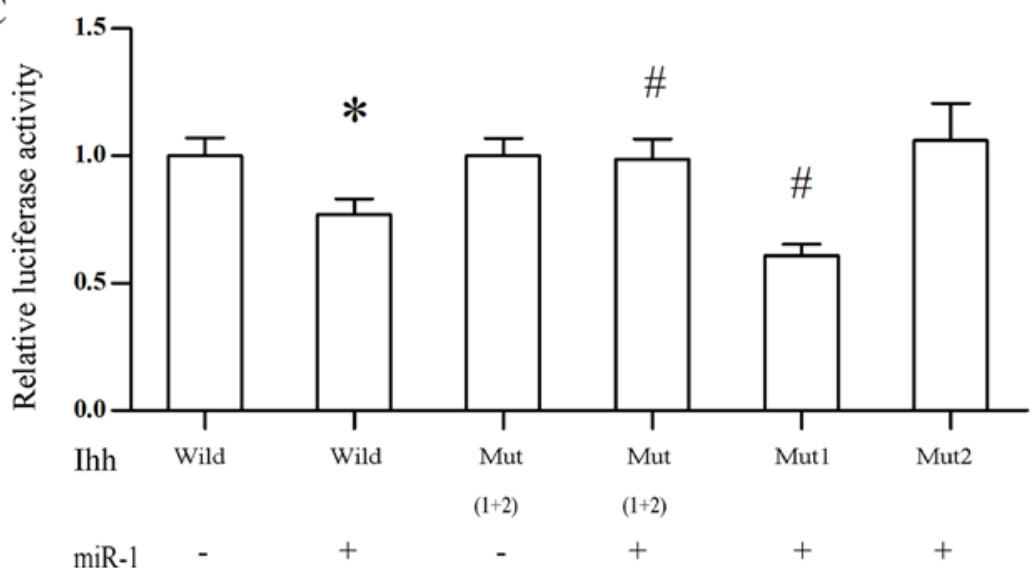

Figure 5. Post-transcriptional regulation of Ihh by miR-1 is dependent on the miR-1 binding site in the 3'UTR of Ihh gene. (A) miR-1 is conserved among different mammalian species. Sequences of the miR-1 target sites in the 3'UTR of Ihh genes of different species are shown. The predicted seeding sites are CATTCCAT and ATGACCTTCCC. (B) Schematic representation of the luciferase vectors with SV40 promoter, the luciferase gene and target fragments in the 3'UTR of human Ihh gene are shown. The desired SV40-Ihh 3'UTR muta(1), SV40-Ihh 3'UTR muta(2) and SV40-Ihh 3'UTR muta(1+2) were confirmed by sequencing. (C) Luciferase activity of the Ihh 3'UTR was analyzed in 293T cells transfected with the luciferase plasmids containing differently mutated binding sites within the Ihh 3'UTR alone or together with the miR-1 mimic $(120 \mathrm{nM})$. At $48 \mathrm{~h}$ after transfection, 293T cells were collected for quantification of dual luciferase activities. ${ }^{*} \mathrm{P}<0.05$ vs. wild-type Ihh $3^{\prime} \mathrm{UTR}$ alone; ${ }^{\text {"P }}<0.05$ vs. wild-type Ihh 3 'UTR + miR-1 mimic. miR/miRNA, microRNA; Ihh, Indian hedgehog signaling molecule; UTR, untranslated region.

degradation of Ihh mRNA. The mutagenesis study with single and combined mutations of the miR-1 binding sites further revealed that the binding region 2 in the 3 'UTR of the Ihh mRNA was required for the miR-1 actions.

This report provided evidence that miR-1 is a critical mediator in regulating chondrocyte proliferation, which is associated with Ihh suppression via the targeting the 3'UTR of Ihh mRNA by miR-1. However, the role of miR-1 in regulating Ihh expression seems to be species-dependent and tissue-dependent. In primary chicken embryonic chondrocytes, the mRNA levels of Ihh and Col-X can be significantly increased at $24 \mathrm{~h}$ after the transfection of the miR-1 mimic (18). Therefore, miR-1 was proposed to induce chondrocyte differentiation. On the contrary, the present study revealed that miR-1 downregulated the expression of Ihh and Col-X in cultured mouse thorax chondrocytes. miR-1 seems to differentially regulate the expression of AGG (33), the major cartilaginous proteoglycan gene, in mouse and human or chicken chondrocytes. Transfection of human chondrocytic HCS-2/8 cells, and chicken normal chondrocytes with miR-1 reduced the expression of AGG, whereas overexpression of miR-1 in culture mouse chondrocytes significantly increased AGG mRNA. Therefore, the specific roles of miR-1 in regulating chondrogenesis under the context of species, development stages, and tissues need to be further investigated.

In summary, the present data indicated that miR-1 is able to regulate cell proliferation and the expression of matrix synthesis and chondrocyte differentiation associated molecules in mouse thorax chondrocytes. miR-1 post-transcriptionally suppressed Ihh expression by binding to the 3'UTR of the Ihh gene, and inhibiting downstream Hedgehog signaling. These findings may provide new insights into the molecular mechanisms of miR-1 in regulating chondrocyte phenotypes and suggest the potential use of a miR-1 mimic to treat chondrocyte hypertrophy. 

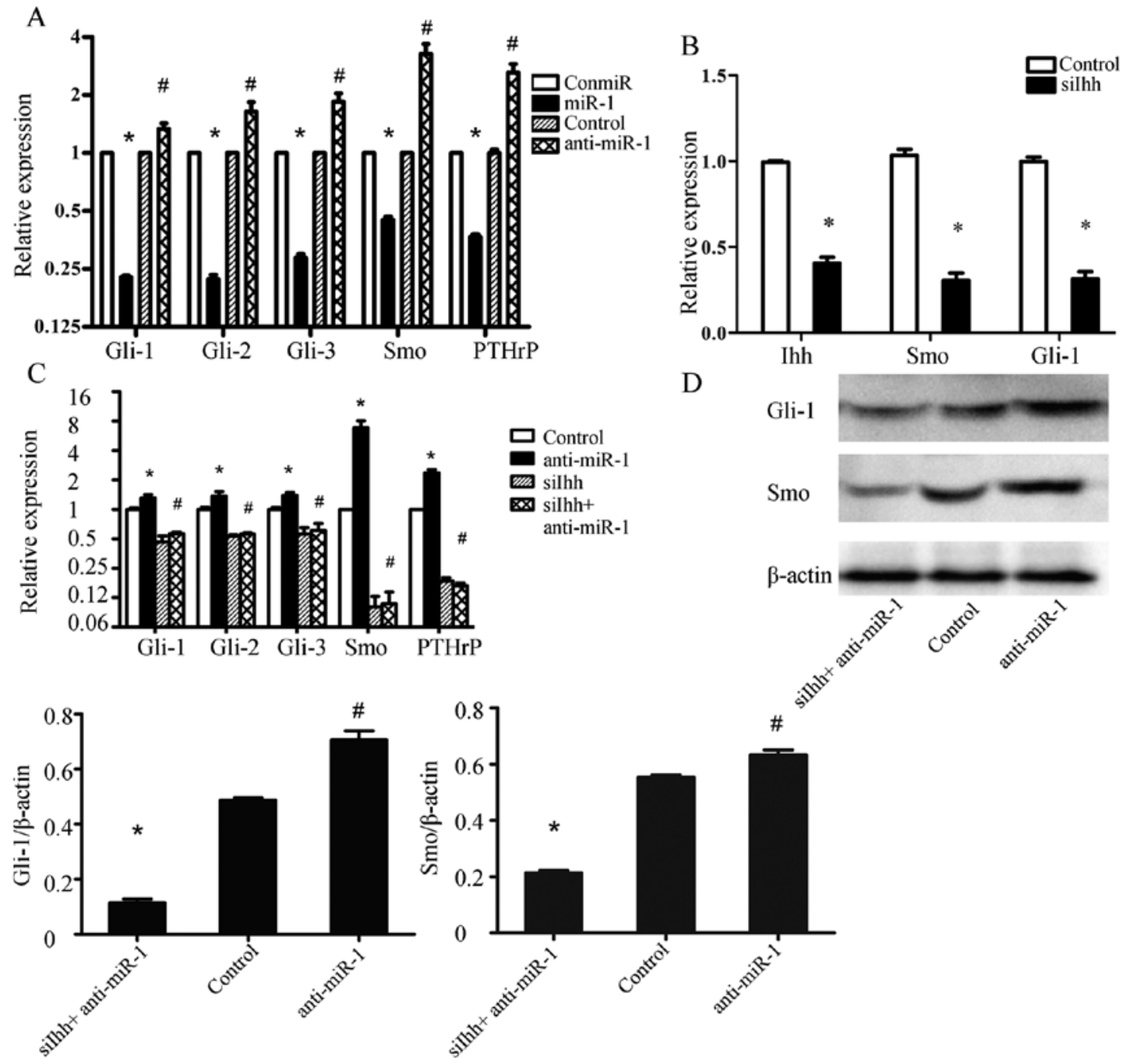

Figure 6. miR-1 decreases the expression of Ihh downstream molecules in the Hedgehog signaling pathway in mouse thorax chondrocytes. (A) Mouse thorax chondrocytes with the indicated transfection were subjected to RT-qPCR in order to analyze mRNA levels of Gli-1, Gli-2, Gli-3, Smo and PTHrP genes at 24 h post-transfection. $\mathrm{n}=6$ for each group; ${ }^{*} \mathrm{P}<0.05$ vs. ConmiR group; ${ }^{*} \mathrm{P}<0.05$ vs. Control group. (B) Knockdown efficiency of siRNA against mouse Ihh in the chondrocytes and the impact of Ihh knockdown on the expression of Ihh downstream genes Smo and Gli-1 were determined by RT-qPCR at $24 \mathrm{~h}$ post-siRNA transfection. $\mathrm{n}=6$ for each group. "P $<0.05$ vs. Control. (C) Mouse thorax chondrocytes with indicated treatments were subjected to RT-qPCR for Gli-1, Gli-2, Gli-3, Smo and PTHrP at 24 h post-siRNA transfection. Control, cells transfected with the control miRNA; Anti-miR-1, cells transfected with the miR-1 inhibitor; silhh, cells transfected with the mouse Ihh-targeted siRNA oligos; siIhh + Anti-miR-1, cells simultaneously transfected the mouse Ihh-targeted siRNA oligos and anti-miR-1. $\mathrm{n}=6$ for each group. ${ }^{*} \mathrm{P}<0.05$ vs. Control group; ${ }^{*} \mathrm{P}<0.05$ vs. silhh group. (D) Protein expression of Gli- 1 and Smo were assessed by western blot assays. The representative images are from one of at least three different experiments with similar results. The histograms show the densitometric analysis of Gli- 1 and Smo expression, with normalization to the $\beta$-actin levels. $n=3$ for each group; ${ }^{*} \mathrm{P}<0.05$ vs. Control group; ${ }^{~} \mathrm{P}<0.05$ anti-miR-1 vs. Control group. miR/miRNA, microRNA; Ihh, Indian hedgehog signaling molecule; RT-qPCR, reverse transcription-quantitative PCR; Gli, GLI family zinc finger; Smo, smoothened, frizzled class receptor; PTHrP, parathyroid hormone-like hormone; siRNA, short interfering RNA.

\section{Acknowledgements}

Not applicable.

\section{Funding}

This study was supported by grants from the National Natural Science Foundation of China (grant no. 81601949), International Cooperation Projects in Shanxi Province (grant no. 201803D421066), Research Project Supported by Shanxi Scholarship Council of China (grant no. 2016-118), Supported by Scientific and Technological Innovation Programs of Higher Education Institutions in Shanxi (grant no. 20161119), Innovation and Entrepreneurship Training Program for College Students in Shanxi Province (grant no. 2018185),
Scientific and Technological Innovation Programs of Shanxi Medical University (grant no. 01201509).

\section{Availability of data and materials}

The datasets used and/or analyzed during the current study are available from the corresponding author on reasonable request.

\section{Authors' contributions}

TC, XC, JL and $\mathrm{ZH}$ performed the experiments, contributed to data analysis and wrote the manuscript. $\mathrm{PH}, \mathrm{CW}$ and $\mathrm{BL}$ analyzed the data. XW, LW and PL conceptualized the study design, contributed to data analysis and revision of the manuscript. All authors read and approved the manuscript and agree 
to be accountable for all aspects of the research in ensuring that the accuracy or integrity of any part of the work are appropriately investigated and resolved.

\section{Ethics approval and consent to participate}

All experiments involving the use of animals in this study were approved by the Ethics Committee of Shanxi Medical University (Taiyuan, Shanxi, China).

\section{Patient consent for publication}

Not applicable.

\section{Competing interests}

The authors declare that they have no competing interests.

\section{References}

1. Rupaimoole R and Slack FJ: MicroRNA therapeutics: Towards a new era for the management of cancer and other diseases. Nat Rev Drug Discov 16: 203-222, 2017.

2. Bagga S, Bracht J, Hunter S, Massirer K, Holtz J, Eachus R and Pasquinelli AE: Regulation by let-7 and lin-4 miRNAs results in target mRNA degradation. Cell 122: 553-563, 2005.

3. Eulalio A, Rehwinkel J, Stricker M, Huntzinger E, Yang SF, Doerks T, Dorner S, Bork P, Boutros $M$ and Izaurralde E: Target-specific requirements for enhancers of decapping in miRNA-mediated gene silencing. Genes Dev 21: 2558-2570, 2007.

4. Valencia-Sanchez MA, Liu J, Hannon GJ and Parker R: Control of translation and mRNA degradation by miRNAs and siRNAs. Genes Dev 20: 515-524, 2006.

5. Liu X, Xiao J, Zhu H, Wei X, Platt C, Damilano F, Xiao C, Bezzerides V, Boström P, Che L, et al: miR-222 is necessary for exercise-induced cardiac growth and protects against pathological cardiac remodeling. Cell Metab 21: 584-595, 2015.

6. Mehta A, Mann M, Zhao JL, Marinov GK, Majumdar D, Garcia-Flores Y, Du X, Erikci E, Chowdhury K and Baltimore D The microRNA-212/132 cluster regulates B cell development by targeting Sox4. J Exp Med 212: 1679-1692, 2015.

7. Guay C, Kruit JK, Rome S, Menoud V, Mulder NL, Jurdzinski A, Mancarella F, Sebastiani G, Donda A, Gonzalez BJ, et al: Lymphocyte-derived exosomal microRNAs promote pancreatic $\beta$ cell death and may contribute to Type 1 diabetes development. Cell Metab 29: 348-361.e6, 2019.

8. Singh PB, Pua HH, Happ HC, Schneider C, von Moltke J, Locksley RM, Baumjohann D and Ansel KM: MicroRNA regulation of type 2 innate lymphoid cell homeostasis and function in allergic inflammation. J Exp Med 214: 3627-3643, 2017.

9. Valenti MT,Dalle Carbonare L and Mottes M: Role of microRNAs in progenitor cell commitment and osteogenic differentiation in health and disease (Review). Int J Mol Med 41: 2441-2449, 2018.

10. Wang Y, Liang Y and Lu Q: MicroRNA epigenetic alterations: Predicting biomarkers and therapeutic targets in human diseases. Clin Genet 74: 307-315, 2008.

11. Goldring MBandMarcuKB:EpigenomicandmicroRNA-mediated regulation in cartilage development, homeostasis, and osteoarthritis. Trends Mol Med 18: 109-118, 2012.

12. Zhang Y, Huang X and Yuan Y: MicroRNA-410 promotes chondrogenic differentiation of human bone marrow mesenchymal stem cells through down-regulating Wnt3a. Am J Transl Res 9: 136-145, 2017.

13. Zhao C, Miao Y, Cao Z, Shi J, Li J, Kang F, Dou C, Xie Z, Xiang Q and Dong S: MicroRNA-29b regulates hypertrophy of murine mesenchymal stem cells induced toward chondrogenesis. J Cell Biochem 120: 8742-8753, 2019.

14. Chen S, Xu Z, Shao J, Fu P and Wu H: MicroRNA-218 promotes early chondrogenesis of mesenchymal stem cells and inhibits later chondrocyte maturation. BMC Biotechnol 19: 6, 2019.

15. Wüst S, Dröse S, Heidler J, Wittig I, Klockner I, Franko A, Bonke E, Günther S, Gärtner U, Boettger T, et al: Metabolic maturation during muscle stem cell differentiation is achieved by miR-1/133a-mediated inhibition of the Dlk1-Dio3 Mega gene cluster. Cell Metab 27: 1026-1039.e6, 2018.
16. Chen JF, Mandel EM, Thomson JM, Wu Q, Callis TE, Hammond SM, Conlon FL and Wang DZ: The role of microRNA-1 and microRNA-133 in skeletal muscle proliferation and differentiation. Nat Genet 38: 228-233, 2006.

17. Zhao Y, Ransom JF, Li A, Vedantham V, von Drehle M, Muth AN, Tsuchihashi T, McManus MT, Schwartz RJ and Srivastava D: Dysregulation of cardiogenesis, cardiac conduction, and cell cycle in mice lacking miRNA-1-2. Cell 129: 303-317, 2007.

18. LiP,Wei X, Guan Y, Chen Q,Zhao T, Sun C and Wei L: MicroRNA-1 regulates chondrocyte phenotype by repressing histone deacetylase 4 during growth plate development. FASEB J 28: 3930-3941, 2014.

19. J Mohler: Requirements for Hedgehog, a segmental polarity gene, in patterning larval and adult cuticle of drosophila. Genetics 120: 1061-1072, 1988

20. Jahan E, Matsumoto A, Rafiq AM, Hashimoto R, Inoue T, Udagawa J, Sekine $\mathbf{J}$ and Otani H: Fetal jaw movement affects Ihh signaling in mandibular condylar cartilage development: The possible role of Ihh as mechanotransduction mediator. Arch Oral Biol 59: 1108-1118, 2014.

21. Zhou J, Wei X and Wei L: Indian Hedgehog, a critical modulator in osteoarthritis, could be a potential therapeutic target for attenuating cartilage degeneration disease. Connect Tissue Res 55: 257-261, 2014.

22. Wei F, Zhou J, Wei X, Zhang J, Fleming BC, Terek R, Pei M, Chen Q, Liu T and Wei L: Activation of Indian hedgehog promotes chondrocyte hypertrophy and upregulation of MMP-13 in human osteoarthritic cartilage. Osteoarthritis Cartilage 20: 755-763, 2012.

23. Ma RS, Zhou ZL, Luo JW, Zhang H and Hou JF: The Ihh signal is essential for regulating proliferation and hypertrophy of cultured chicken chondrocytes. Comp Biochem Physiol B Biochem Mol Biol 166: 117-122, 2013.

24. Chen X, Macica CM, Nasiri A and Broadus AE: Regulation of articular chondrocyte proliferation and differentiation by indian hedgehog and parathyroid hormone-related protein in mice. Arthritis Rheum 58: 3788-3797, 2008

25. Deng A, Zhang H, Hu M, Liu S, Wang Y, Gao Q and Guo C: The inhibitory roles of Ihh downregulation on chondrocyte growth and differentiation. Exp Ther Med 15: 789-794, 2018.

26. Kurio N, Saunders C, Bechtold TE, Salhab I, Nah HD, Sinha S, Billings PC, Pacifici M and Koyama E: Roles of Ihh signaling in chondroprogenitor function in postnatal condylar cartilage. Matrix Biol 67: 15-31, 2018.

27. Mirando AJ, Dong Y, Kim J and Hilton MJ: Isolation and culture of murine primary chondrocytes. Methods Mol Biol 1130: 267-277, 2014.

28. Wei L, Fleming BC, Sun X, Teeple E, Wu W, Jay GD, Elsaid KA, Luo J, Machan JT and Chen Q: Comparison of differential biomarkers of osteoarthritis with and without posttraumatic injury in the Hartley guinea pig model. J Orthop Res 28: 900-906, 2010.

29. Livak KJ and Schmittgen TD: Analysis of relative gene expression data using real-time quantitative PCR and the 2(-Delta Delta C(T)) method. Methods 25: 402-408, 2001.

30. Guan Y, Chen Q, Yang X, Haines P, Pei M, Terek R, Wei X, Zhao T and Wei L: Subcellular relocation of histone deacetylase 4 regulates growth plate chondrocyte differentiation through $\mathrm{Ca}^{2+} / \mathrm{calmodulin}$-dependent kinase IV. Am J Physiol Cell Physiol 303: C33-C40, 2012.

31. Wei L, Kanbe K, Lee M, Wei X, Pei M, Sun X, Terek R and Chen Q: Stimulation of chondrocyte hypertrophy by chemokine stromal cell-derived factor 1 in the chondro-osseous junction during endochondral bone formation. Dev Biol 341: 236-245, 2010.

32. Zhou J, Chen Q, Lanske B, Fleming BC, Terek R, Wei X, Zhang G, Wang S, Li K and Wei L: Disrupting the Indian hedgehog signaling pathway in vivo attenuates surgically induced osteoarthritis progression in Col2a1-CreERT2; Ihhfl/fl mice. Arthritis Res Ther 16: R11, 2014.

33. Sumiyoshi K, Kubota S, Ohgawara T, Kawata K, Nishida T, Shimo T, Yamashiro T and Takigawa M: Identification of miR-1 as a micro RNA that supports late-stage differentiation of growth cartilage cells. Biochem Biophys Res Commun 402: 286-290, 2010.

34. Leung VY, Gao B, Leung KK, Melhado IG, Wynn SL, Au TY, Dung NW, Lau JY, Mak AC, Chan D, et al: SOX9 governs differentiation stage-specific gene expression in growth plate chondrocytes via direct concomitant transactivation and repression. PLoS Genet 7: e1002356, 2011.

This work is licensed under a Creative Commons Attribution-NonCommercial-NoDerivatives 4.0 International (CC BY-NC-ND 4.0) License. 